be a presenting feature of underlying lymphoreticular malignancy. ${ }^{2}$ Ilfred $e t$ al have also reported four cases of benign monoclonal gammopathy associated with PMR and temporal arteritis (TA). ${ }^{3}$ Sera from these patients were found to contain heavy or light chains which bound to mouse femoral artery and which corresponded to the heavy and ligh chains of the patients' benign monoclonal gammopathy. They postulated that the benign monoclonal gammopathy may be anti-artery antibodies, possibly associated with the pathogenesis of PMR or TA.

These cases emphasise the need to remain aware of additional causes of a raised ESR in patients who present with PMR/TA. We agree that a chest X-ray and serum and urine electrophoresis should form part of the routine investigation of PMR/TA.

EP DAS-GUPTA P BANDYOPADHYAY JL KOK SHUN JP NG

Department of Haematology Barnsley District General Hospital NHS Trust Barnsley, South Yorks S75 2EP, UK

1 Speed CA, Haslock I. Polymyalgia rheumatica, temporal arteritis and malignancy. Postgrad Med F 1995; 71: $500-1$.

2 Kalra L, Delamere JP. Lymphoreticular malignancy and monoclonal gammopathy presenting as polymyalgia rheumatica. $B r \mathcal{F}$ Rheumatol 1987 , 26: $458-9$.

3 Ilfield D, Barzilay J, Vana D, Ben-Bassat $M$ Joshua H, Pick I. IgG monoclonal gammopathy in four patients with polymyalgia rheumatica. Ann Rheum Dis 1985; 44: 501 -2.

\section{Management of acute myocardial infarction}

Sir

I read with interest the excellent review by Saltissi and Mushahwar on 'The managemen of acute myocardial infarction'. ${ }^{1}$ They discussed the value of thrombolysis given late, ie, more than 12 hours after the onset of pain. However they did not comment on the importance of the site of infarction. Patients with inferior myocardial infarction benefit less from thrombolysis than those with anterio infarction ${ }^{2}$ as their overall mortality is lower. Some even question the evidence for giving thrombolysis at all in this group. ${ }^{3}$ Studies have been undertaken to assess the possible benefit of late thrombolysis. ${ }^{4,5}$ Overall no significant benefits have been shown later than 12 hours. Subgroup analysis, however, has suggested that there may be groups that do benefit but the identification of these individuals is not possible on current data. Saltissi and Mushahwar recommended that each patient presenting at or after 12 hours be treated on their own merits. Site of infarction should surely be a major consideration?

M TURNER

Department of Cardiology, Derriford Hospital, Plymouth, Devon, UK

1 Saltissi S, Mushahwar. The management of acute myocardial infarction. Postgrad Med $\mathfrak{f}$ 1995; 71: 534-41.

2 Fibrinolytic Therapy Trialists' (FTT) Collaborative Group. Indications for fibrinolytic therapy i suspected acute myocardial infarction: collaborative overview of early mortality and major morbidity results from all randomised trials of more than 1000 patients. Lancet 1994; 343: $311-21$.

3 Tobe TJM. Is thrombolytic therapy in acute inferior myocardial infarction really better than conventional treatment? $\mathrm{Br}$ Heart $\mathcal{f}$ 1995; 73: conven.

4 LATE Study Group. Late assessment of thrombolytic efficacy (LATE) study with alteplase 6 24 hours after onset of acute myocardia infarction. Lancet 1993; 342: 759-66.

5 EMERAS Collaborative Group. Randomised trial of late thrombolysis in patients with suspected acute myocardial infarction. Lancet 1993; 342: 767-72.

This letter was shown to Drs Saltissi and Mushahwar who responded as follows:

Sir

Turner raises the vexed question of the effect of site of infarction (inferior versus anterior) on the efficacy of thrombolysis and whether this criterion should be used in deciding on the need for treatment in an individual patient.

In a review of limited size covering an enormous clinical area, we opted to omit discussion of the effect of infarct site because the jury remains out on this question. To our knowledge, although the large randomised trials of fibrinolytic therapy report baseline characteristics with regard to infarct site none has randomised patients prospectively on that basis. Retrospective subgroup analysis has usually shown a large significant benefit in anterior infarcts and a smaller, borderline non significant effect in inferior infarcts. The dangers of retrospective subgroup analysis are, however, well recognised and the results of such analyses should be used to generate hypotheses for testing in future trials rather than to answer questions in their own right.

Even systematic overview (meta-analysis) does not necessarily eliminate the statistical problems and give reliable results. The accuracy of analysis depends on the uniformity of the trials included, the more hetero- genous the studies the more unreliable the result. Thus, the overview of effects of fibrinolytic treatment by the Fibrinolytic Therapy Trialist's Collaborative Group (ref 2 above) included all trials in which more than 1000 patients were randomised between fibrinolytic therapy and control in the absence of prior knowledge of the next treatment and unconfounded by other treatment variables. However, these trials varied enormously in other ways, eg, fibrinolytic agent used, use of adjunctive heparin and aspirin, time of randomisation, entry ECG criteria, etc. Thus a degree of uncertainty about the ensuing results must remain. Despite this, a strong trend to benefit from treatment by thrombolytic agents in inferior infarcts was found. They estimated that eight lives would be saved per 1000 patients treated which compares favourably with the measured detriment of two additional fatal strokes and three bleeds per 1000 patients treated.

A recent personal viewpoint (ref 3 above), cast doubt on the efficacy of thrombolytic therapy in inferior infarction. The author proposed several possible pathophysiological reasons why there might be a lesser benefit in inferior infarction. However the history of medicine is littered with treatments which seem a good idea at the time but which did not stand up to rigorous examination. Furthermore, the author recognised the pitfalls of the retrospective subgroup analysis used in his article. In our view, it is not acceptable to acknowledge such pitfalls and then dismiss them on the basis of untested pathophysiological hypotheses. To our mind, questionable science and plausable but unproven hypotheses neither singly nor together constitute good grounds for deciding on treatment guidelines.

Turner also alludes to the benefit of late thombolysis and implies that site of infarction should be considered in deciding which subgroups might benefit. Two studies, LATE and EMERAS (refs 4,5 above) have specifically looked at the value of late thombolysis. Both stated the baseline proportion of patients with anterior and inferior infarction but, to our knowledge, neither prospectively randomised nor reported results on the basis of site of infarction. In our view it is therefore not possible to comment reliably on effects of site of infarction on the efficacy of thrombolysis delivered in the time windows $6-12$ hours or 12-24 hours post-infarct.

SALTISSI $S$ MUSHAHWAR

Royal Liverpool and Broadgreen University Hospitals NHS Trust, Prescot Street, Liverpool L7 8XP, UK

The Editor would like to congratulate the winners who will each receive a free subscription to the Postgraduate Medical fournal for 1996 